\title{
MiRNAs involved in the signaling pathways associated to the pathogenesis of idiopathic pulmonary fibrosis
}

\author{
Ana R. Cadena-Suárez* \\ Molecular Biology Laboratory "Ismael Cosío Villegas" National Institute of Respiratory Diseases, Mexico City, Mexico
}

\begin{abstract}
Idiopathic Pulmonary Fibrosis (IPF) is a progressive and multifactorial interstitial lung disease whose pathophysiology remains unclear. The process initiates by repeated epithelial lung injuries followed by basal membrane destruction occasioning the activation of the epithelial mesenchymal transition (EMT) and myofibroblasts which carry out an excessive synthesis of extracellular matrix (ECM) proteins. Several studies have associated microRNAs (miRNAs) to the biogenesis and development of IPF because miRNAs participate in the processes of apoptosis, proliferation, differentiation and interaction between cells thanks to their role as activators or inhibitors of different receptors in the signaling pathways of TGF- $\beta$, Wnt/ $\beta$-catenin and PI3K-Akt-FOXO3a-mTOR, which are the main and most studied pathways involved in lung fibrosis. In this context, the knowledge on the altered miRNAs expression and even more their role in the different signaling pathways in IPF will shed light on new therapeutic targets for this lung disease.
\end{abstract}

Keywords: miRNAs. Idiopathic pulmonary fibrosis. Epithelial mesenchymal transition. Myofibroblasts.

\section{Introduction}

Idiopathic pulmonary fibrosis (IPF) is a chronic, progressive disease with poor prognosis, and $f$ ew therapeutic options. The incidence and prevalence increase with age, and it is most common seen in male patients older than 65 years. At first, IPF was considered as a chronic inflammatory disorder characterized by progressive fibrosis, however, evidence indicate that this pathology is a product of an epithelial-driven disorder associated with environmental, genetic risk factors, aging-associated processes and a profibrotic epigenetic reprogramming ${ }^{1}$. During the development of IPF, lung parenchyma shows repeated epithelial cell injuries with an aberrant reparation mechanism that cause several lung scars, mainly caused by an excessive fibroblasts activity and their trans differentiation to myofibroblasts. Moreover, the epithelial-mesenchymal transition (EMT) promotes myofibroblasts accumulation, and synthesis of an excessive amount of collagen and extracellular matrix (ECM) proteins by the alveolar epithelial cells (AECs) ${ }^{2}$.

MiRNAs are short, non-coding RNAs with an extension of approximately 21-23 nucleotides which play a post-transcriptional regulatory role by targeting specific messenger RNAs for degradation and/or translational repression. To date, there are already some reports about profibrotic and anti-fibrotic miRNAs dysregulation in the pathogenesis of IPF. These mechanisms are carried out since miRNAs have overlapping functions because they can either regulate a single gene or several genes can be affected by a single miRNA. For this reason, is relevant to update the information about altered miRNAs involved in the crucial signaling pathways implicated in IPF to have a better understanding of their pathogenesis mechanisms and to propose possible potential therapeutic targets ${ }^{3}$.

\section{Correspondence:}

*Ana R. Cadena-Suárez

E-mail: anah.cdn@gmail.com
Available online: 07-03-2022

Date of reception: 29-06-2021

Date of acceptance: 30-09-2021

DOI: 10.24875/HGMX.21000049
Rev Med Hosp Gen Mex. 2022;85(1):34-43 www.hospitalgeneral.mx

0185-1063/@ 2021 Sociedad Médica del Hospital General de Mexico. Published by Permanyer. This is an open access article under the CC BYNC-ND license (http://creativecommons.org/licenses/by-nc-nd/4.0/). 


\section{Materials and Methods}

This review is focused on a PubMed searching using the following key words: "miRNAs involved in IPF", "miRNAs involved in cellular signaling pathways in IPF" and "miRNAs involved in the TGF- $\beta$ pathway". The selection was reduced to articles written in the years 2016 to 2020 in English language. The articles found were around fifty; however, the selected papers were the ones that related miRNAs with the TGF- $\beta$, Wnt/ $\beta$-catenin and PI3K-Akt-FOXO3a-mTOR signaling pathways since these signaling pathways are the most studied ones in pulmonary fibrosis process.

For better understanding, the manuscript is organized according to miRNAs participation in each signaling pathway involved in the pathogenesis in IPF.

First, a general summary about the main signaling pathways involved in the pathogenesis of IPF is explained and then there is a description of the function of each miRNA within them.

\section{EMT process in IPF}

EMT is a process in which epithelial cells gradually lose their cell-to-cell junctions, adherens junctions and apical-basal polarity to acquire a mesenchymal cell phenotype. This phenotype gains new invasive properties, and re-synthesizing new extracellular matrix proteins. The EMT process, can be activated by transcription factors and by signaling pathways, being the transforming growth factor beta 1 (TGF- $\beta$ ) its best inductor ${ }^{4}$. This process is controlled by three families of transcription factors: the zinc finger Snail (Snail/Slug) and zinc finger E-Box binding homeobox 1 and 2 (ZEB1/ZEB2) which have, as main function, the repression of E-cadherin expression $^{5}$. Nowadays, it is well stablished that EMT process in IPF is regulated also, by epigenetic modulators like miRNAs which can act by activating or reverting lung fibrosis through the regulation of targets expression involved in EMT signaling pathways such as TGF- $\beta$, Wnt/B-catenin and PI3K-Akt-FOXO3a-mTOR, which induce the activation of resident fibroblasts and their trans differentiation into myofibroblasts, for example, miRNA-326 reduces the expression of profibrotic genes by targetting TGF- $\beta$ and miRNA-26 directly downregulates CTGF which is the responsable for collagen formation ${ }^{6}$.

\section{Signaling pathways involved in EMT process}

EMT is regulated by extracellular ligands such as TGF- $\beta$, interleukin-1 (IL-1), connective tissue growth factor (CTGF) and nuclear factor kappa-light-chain-enhancer of activated B cells (NF-kb), which is activated by tumor necrosis factor alpha (TNF- $\alpha$ ), insulin-like growth factor-1 (IGF-1), secreted glycoproteins of the wingless/integrate-1 (Wnt) ${ }^{5}$ and ADAM metallopeptidase with thrombospondin type motif 1 (ADAMTS1).

All of them, have the property of initiating the intracellular signaling cascades through the activation of one or more EMT-driving transcription factors such as SNAIL1/2 and ZEB1/2 and downregulating the expression of adhesion molecules like E-cadherin, being the high-mobility group AT-hook2 (HMGA2) the one that facilitates the transcription of SNAIL1 ${ }^{5}$.

It has also been proved that MiRNAs have a stimulant role in extracellular matrix protein synthesis through collagen genes such as COL1A1, COL1A2, COL3A1, COL5A2, COL4A2, LOXL and matrix metallopeptidase (MMP) like MMP2, MMP3 and MMP10, among others ${ }^{8}$.

\section{TGF-ß signaling pathway}

In lung diseases like IPF, there is an increasing evidence that proves the activation of TGF- $\beta$ pathway by lung cells to maintain homeostasis in response to tissue injury ${ }^{9}$. This cytokine is secreted, in a latent form, bound to latency-associated peptide (LAP), but when it is released from this peptide, TFG- $\beta$ dimers conform a complex with the type I TGF- $\beta$ receptor (TGF-BRI) and type II receptor (TGF-BRII). This complex initiates a signal transduction by a Smad-dependent or non-Smad-dependent pathway ${ }^{8}$. In the Smad-dependent pathway, activated TGF-BRI phosphorylates cytoplasmic Smad2/3 transcription factors and translocate them into the nucleus. Smad4 facilitate this process and together with ZEB1/2 complex modulate the pro-fibrotic genes expression associated with EMT process, fibroblasts differentiation, proliferation migration and deposition of collagen ${ }^{10}$. Moreover, the Smad 2/3 complex represses the expression of E-cadherin through SNAIL1 and SNAIL2 which induce the expression of mesenchymal proteins such as $\mathrm{N}$-cadherin, fibronectin, and metalloproteinases ${ }^{11}$. On the other hand, Smad6 and Smad7 inhibits the phosphorylation of Smad 2/3 complex by competing with Smad4 or TGF- $\beta$ RI which is also regulated by Smurf2 E3 ubiquitin ligase. For this way, TGF- $\beta$ signaling pathway is downregulated by Smad6, Smad7, and Smurf2 ${ }^{10}$.

In the non-Smad mediated pathway TGF- $\beta$ can activate other signaling cascades, like mitogen-activated protein kinase (MAPK) pathway which can mediate Smad phosphorylation and activation through the 
activation of extracellular signal-regulated kinase (Erk) and C-Jun N-terminal kinase (JNK) pathways. Also, TGF- $\beta$ can activate other signaling mediators such as p38 MAPK kinase kinases family (MAPKKKS) and TGF- $\beta$ activated kinase 1 (TAK1) which has the function of activating NF-kB signaling pathway. This dual ability of TGF- $\beta$ to activate Smad and non Smad signaling pathways has an important role in the EMT process $^{12}$.

There are many others signaling pathways involved in the pathogenesis of IPF, however, the Wnt/ $\beta$-catenin and phosphoinositide 3 kinase (PI3K)/protein kinase $B$ (Akt) (PI3K/Akt) signaling pathways are the most relevant because they are related to cell proliferation, differentiation, and extracellular matrix production.

\section{Wnt/ $/$ catenin signaling pathway}

In presence of injury Wnt/ $\beta$-catenin signaling pathway promotes alveolar repair and remodeling, fibroblast migration and proliferation and extracellular matrix production through the stimulation of $\beta$-catenin by $\mathrm{Wnt}^{13}$.

The activation of this pathway begins with the stimulation of TGF- $\beta$ which generates the release of Wnt from the axis inhibition protein 1 (AXIN1)- glycogen synthase kinase 3 beta (GSK3 $\beta$ ) - adenomatous polyposis coli (APC) (AXIN1/GSK3 $\beta / A P C)$ complex, which, in a steady state, leads to ubiquitin-mediated proteolysis of $\beta$-catenin. When $W n t$ is released from this complex, there is a nuclear translocation of $\beta$-catenin via the phosphorylation of extracellular signal-regulated kinases 1 and 2 (ERK1/2) and suppression of GSK-3b and AXIN1 activity, this causes the accumulation of $\beta$-catenin and then suffers a nuclear translocation, by this way it can regulate the fibronectin gene 1 (FN1), the metalloproteinase 7 (Mmp 7) and cyclin D1 with the consequent production of extracellular matrix ${ }^{14}$.

\section{PI3K-Akt signaling pathway}

This pathway has the function of regulating fibroblasts growth, proliferation, apoptosis, and collagen production; however, this pathway is down regulated in IPF which leads to Akt up regulation under mechanisms that modulate the activity of the phosphatidylinositol 3-kinase (PI3K)-protein kinase B (Akt)- forkhead box $\mathrm{O} 3$ (FoxO3a) and mammalian target of rapamycin (mTOR) (PI3K-Akt-FOXO3a-mTOR) axis. PI3K stimulates the synthesis of phosphatidylinositol-3,4,5-triphosphate (PIP3) which causes the activation of Akt. One of Akt targets is FoxO3a which is a powerful inhibitor of cell cycle due to the activation of the cyclin-dependent kinases (CDK) inhibitor protein p27 which promotes $\mathrm{G} 1$ arrest, however, FoxO3a activity is abnormally low in IPF fibroblasts because phosphatase and tensin homolog (PTEN), the main regulator of this pathway, is poorly expressed. This leads to fibroblasts autophagy inhibition thus promoting fibroblasts proliferation, increases the expression of alpha-smooth muscle actin ( $\alpha$-SMA) and fibronectin and reduces collagen degradation creating a collagen-rich environment ${ }^{15}$.

MTOR has a role in cell growth, proliferation and apoptosis, it forms two distinct complexes which are mammalian target of rapamycin complex 1 (mTORC1) and mammalian target of rapamycin complex 2 (mTORC2), the first one controls cell growth and the second one regulates cell proliferation and survival: being the tuberous Sclerosis complex (TSC) the negative regulator of $\mathrm{mTORC} 1$ and $\mathrm{PI} 3 \mathrm{~K}$ the negative regulator of mTORC2 ${ }^{16}$.

MiRNAs have an important role in these signaling pathways because they can act as pro fibrotic or antifibrotic mediators through the stimulation or inactivation of different receptors in the signaling cascade, further examples of this will be seen below.

Figure 1 summarizes the different signaling pathways mentioned above, such as TGF- $\beta$, Wnt/ $\beta$-catenin and PI3K-Akt-FOXO3a-mTOR as well as their cross-linking. Also, several receptors involved in these pathways are illustrated, which can be stimulated or inhibited by the action of different miRNAs which can either stimulate or inhibit the trans differentiation of fibroblasts into myofibroblasts, pro fibrotic genes activation and extracellular matrix proteins synthesis.

\section{MiRNAs involved in the pathogenesis of IPF}

The compilation of miRNAs implicated in IPF was grouped depending on the signaling route and the process they regulate. They are better illustrated in Table 1.

\section{MiRNAs involved in EMT in a Smad- dependent way}

There are some miRNAs that have been studied in different samples like in a bleomycin murine model, in human A549 cells, bronchial epithelial cells and mice fibroblasts respectively for their antifibrotic role in IPF due to their up regulation, such as miRNA-877-3p, miRNA-1343 and miRNA-133. 


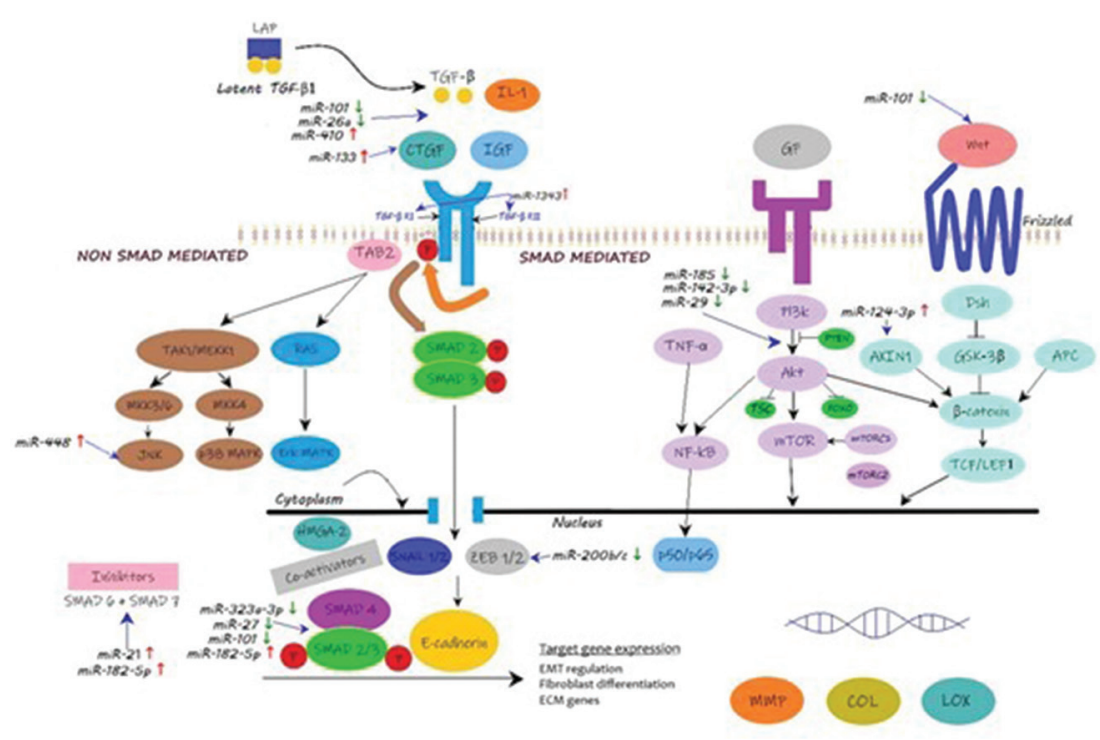

Figure 1. Proposal crosstalk between TGF- $\beta$ with Smad, non-Smad dependent pathway, Wnt/ $\beta$-catenin and PI3K-Akt-FOX03a-mTOR signaling pathways involved in the EMT process and some examples of miRNAs mentioned in this review involved in the fibrotic process. (Image inspired in the work of Derynck, R., \& Zhang, Y.E. [2003], Rajasekaran, S., Rajaguru, P., \& Sudhakar Gandhi, P. S. [2015] and Razali, R. A., Lokanathan, Y., Yazid, M. D., Ansari, A. S., Saim, A. B., \& Hj Idrus, R. B. [2019]).

MiRNA-877-3p can inhibit TGF- $\beta$-induced myofibroblast differentiation through the up regulation of Smad7 $7^{17}$, miRNA-1343 represses both TGF- $\beta$ I and II receptor thus reducing the expression of fibrosis markers such as $\alpha$-SMA and COL1A ${ }^{18}$ and miRNA-133 inhibits myofibroblast differentiation through the down regulation of profibrotic genes such as COL1A1 and CTGF $^{19}$.

On the other hand, there are miRNAs that even though have an antifibrotic role in health, they favor the development of IPF due to their downregulation status in this disease such as, miRNA-184, miRNA-155 miRNA-27a-3p, miRNA-19a- 19b-20a sub-cluster, miRNA-29b, miRNA-18a-5p, miRNA-200b/c, miRNA-27b, miRNA-185, miRNA-186, miRNA-130b-3p, miRNA-101, miRNA-323a-3p and miRNA-221.

MiRNA-184, studied in A549 cells, is not able to regulate Akt and Smad2 ${ }^{20}$, miRNA-155 studied in human lung fibroblasts, is not able to regulate Smad1 ${ }^{21}$ and miRNA-27a-3p studied in a pulmonary fibroblasts line can not inhibit, the phenotypic marker of myofibroblasts, $\alpha$-SMA, Smad2 and Smad422. These three miRNAs generate an induction of EMT transition, fibroblast proliferation and migration and collagen synthesis.
The next miRNAs have been studied in a bleomycin murine model: MiRNA-19a-19b-20a sub-cluster is not able to suppress pro-fibrotic genes, including COL1A1 and $\mathrm{CTGF}^{23}$, miRNA-29b is not able to suppress the expression COL1A1 and COL3A ${ }^{24}$ and miRNA-18a-5p contributes with EMT because it is not able to regulate TGF- $\beta$ type II receptor which might therefore provide a novel approach to the treatment of IPF ${ }^{25}$. MiRNA-200b/c is a possible therapeutic target too because this miRNA attenuates early pulmonary fibrosis by inhibiting ZEB1/2 via Smad-mediated way with the consequent inhibition of the expression of E-cadherin, however its expression is diminished in a fibrosis status ${ }^{26}$, and MiRNA-27b role is affected because it cannot inhibit the expression of collagens genes such as COL1A1, COL3A1, COL4A1 and $\alpha$-SMA through the regulation of Smad2, that is the reason why it has been proposed as a possible therapeutic target as well27.

MiRNA-185, miRNA-186 and miRNA-130b-3p have been studied on human lung fibrotic samples and it has been seen that both miRNA-185 and miRNA-186 fail on the regulation of COL5A1 gene ${ }^{28}$ and miRNA-130b-3p is unable to regulate the action of IGF-1 on EMT process $^{29}$. MiRNA-101 and miRNA-323a-3p have been studied human lung fibrotic samples but also in a 
Table 1. MiRNAs involved in the crucial signaling pathways of Idiopathic Pulmonary Fibrosis (Continued)

\begin{tabular}{|c|c|c|c|c|c|c|c|}
\hline & Sample & miRNA & $\begin{array}{l}\text { Function in } \\
\text { health }\end{array}$ & $\begin{array}{l}\text { Target of } \\
\text { action }\end{array}$ & Effect & $\begin{array}{l}\text { Status in } \\
\text { IPF }\end{array}$ & Reference \\
\hline \multicolumn{8}{|c|}{ MiRNAs involved in the EMT in a Smad-dependent pathway } \\
\hline I & $\begin{array}{l}\text { Bleomycin murine } \\
\text { model }\end{array}$ & miRNA-877-3p & $\begin{array}{l}\text { Anti- } \\
\text { fibrotic }\end{array}$ & Smad7 & $\begin{array}{l}\text { Regulation of } \\
\text { fibroblasts } \\
\text { differentiation into } \\
\text { myofibroblasts }\end{array}$ & $\begin{array}{l}\text { Up } \\
\text { regulated }\end{array}$ & $\begin{array}{l}\text { Wang et al., } \\
2016\end{array}$ \\
\hline II & $\begin{array}{l}\text { Human A549 and } \\
\text { bronchial epithelial } \\
\text { cells }\end{array}$ & miRNA-1343 & $\begin{array}{l}\text { Anti- } \\
\text { fibrotic }\end{array}$ & $\begin{array}{l}\text { TGFBRI and } \\
\text { TGFBRII }\end{array}$ & $\begin{array}{l}\text { Inhibits Smad } 2 / 3 \\
\text { phosphorylation, } \\
\text { nuclear translocation, } \\
\text { and reduces } \\
\text { expression of markers } \\
\text { of fibrosis such as } \\
\alpha-S M A \text { and COL1A1 }\end{array}$ & $\begin{array}{l}\text { Up } \\
\text { regulated }\end{array}$ & $\begin{array}{l}\text { Stolzenburg } \\
\text { et al., } 2016\end{array}$ \\
\hline III & $\begin{array}{l}\text { Mice fibroblasts } \\
\text { NIH3T3 }\end{array}$ & miRNA-133a & $\begin{array}{l}\text { Anti- } \\
\text { fibrotic }\end{array}$ & $\begin{array}{l}\text { COL1A1 gene } \\
\text { and CTGF }\end{array}$ & $\begin{array}{l}\text { Regulation of } \\
\text { TGF- } \beta \text { linduced } \\
\text { myofibroblast } \\
\text { differentiation }\end{array}$ & $\begin{array}{l}\text { Up } \\
\text { regulated }\end{array}$ & $\begin{array}{l}\text { Wei et al., } \\
2019\end{array}$ \\
\hline IV & A549 cells & miRNA-184 & $\begin{array}{l}\text { Anti- } \\
\text { fibrotic }\end{array}$ & $\begin{array}{l}\text { AKT and } \\
\text { Smad2 }\end{array}$ & $\begin{array}{l}\text { Increase of TGF- } \beta \text { and } \\
\text { PI3K-AKT-NF- } \mathrm{B} \\
\text { pathway }\end{array}$ & $\begin{array}{l}\text { Down } \\
\text { regulated }\end{array}$ & $\begin{array}{l}\text { Wang et al, } \\
2020\end{array}$ \\
\hline V & $\begin{array}{l}\text { Human lung } \\
\text { fibroblasts }\end{array}$ & miRNA-155 & $\begin{array}{l}\text { Anti- } \\
\text { fibrotic }\end{array}$ & Smad1 & $\begin{array}{l}\text { Regulation of fibroblast } \\
\text { proliferation, migration, } \\
\text { and collagen synthesis }\end{array}$ & $\begin{array}{l}\text { Down } \\
\text { regulated }\end{array}$ & $\begin{array}{l}\text { Chi et al., } \\
2019\end{array}$ \\
\hline VI & $\begin{array}{l}\text { Human HEK-293T } \\
\text { and pulmonary } \\
\text { fibroblast line }\end{array}$ & miRNA-27a-3p & $\begin{array}{l}\text { Anti- } \\
\text { fibrotic }\end{array}$ & $\begin{array}{l}\text { Smad2 and } \\
\text { Smad4 }\end{array}$ & $\begin{array}{l}\text { Regulation of } \\
\text { myofibroblast } \\
\text { differentiation and } \\
\text { expression of type } 1 \\
\text { collagen, fibronectin } \\
\text { and } \alpha \text {-SMA }\end{array}$ & $\begin{array}{l}\text { Down } \\
\text { regulated }\end{array}$ & $\begin{array}{l}\text { Cui et al., } \\
2016\end{array}$ \\
\hline VII & $\begin{array}{l}\text { Bleomycin murine } \\
\text { model }\end{array}$ & $\begin{array}{l}\text { miRNA-19a- } \\
\text { 19b-20a } \\
\text { sub-cluster }\end{array}$ & $\begin{array}{l}\text { Anti- } \\
\text { fibrotic }\end{array}$ & $\begin{array}{l}\text { TGF-BRI and } \\
\text { TGF-BRII }\end{array}$ & $\begin{array}{l}\text { Regulation of } \\
\text { pro-fibrotic genes, } \\
\text { including } \\
\text { COL1A1 and CTGF }\end{array}$ & $\begin{array}{l}\text { Down } \\
\text { regulated }\end{array}$ & $\begin{array}{l}\text { Souma } \\
\text { et al., } 2018\end{array}$ \\
\hline VIII & $\begin{array}{l}\text { Bleomycin murine } \\
\text { model and NIH/3T3 } \\
\text { cells }\end{array}$ & miRNA-29b & $\begin{array}{l}\text { Anti- } \\
\text { fibrotic }\end{array}$ & $\begin{array}{l}\text { COL1A1 and } \\
\text { COL3A1 }\end{array}$ & $\begin{array}{l}\text { Collagen expression } \\
\text { Regulation }\end{array}$ & $\begin{array}{l}\text { Down } \\
\text { regulated }\end{array}$ & $\begin{array}{l}\text { Yamada } \\
\text { et al., } 2017\end{array}$ \\
\hline IX & $\begin{array}{l}\text { Bleomycin murine } \\
\text { model }\end{array}$ & miRNA-18a-5p & $\begin{array}{l}\text { Anti- } \\
\text { fibrotic }\end{array}$ & TGF-BRII & EMT regulation & $\begin{array}{l}\text { Down } \\
\text { regulated }\end{array}$ & $\begin{array}{l}\text { Zhang } \\
\text { et al., } 2017\end{array}$ \\
\hline$X$ & $\begin{array}{l}\text { Bleomycin murine } \\
\text { model }\end{array}$ & miRNA-200b/c & $\begin{array}{l}\text { Anti- } \\
\text { fibrotic }\end{array}$ & ZEB1 and ZEB2 & $\begin{array}{l}\text { Regulation of } \\
\text { E-cadherin expression } \\
\text { and EMT }\end{array}$ & $\begin{array}{l}\text { Down } \\
\text { regulated }\end{array}$ & $\begin{array}{l}\text { Cao et al., } \\
2018\end{array}$ \\
\hline $\mathrm{XI}$ & $\begin{array}{l}\text { Bleomycin murine } \\
\text { model }\end{array}$ & miRNA-27b & $\begin{array}{l}\text { Anti- } \\
\text { fibrotic }\end{array}$ & $\begin{array}{l}\text { TGFBR1 and } \\
\text { Smad2 }\end{array}$ & $\begin{array}{l}\text { Regulation of } \\
\text { fibroblasts activation } \\
\text { and expression of } \\
\text { COL1A1, COL3A1, } \\
\text { COL4A1 and } \alpha-\text { SMA }\end{array}$ & $\begin{array}{l}\text { Down } \\
\text { regulated }\end{array}$ & $\begin{array}{l}\text { Zeng et al., } \\
2017\end{array}$ \\
\hline XII & $\begin{array}{l}\text { Human lung } \\
\text { fibrotic samples }\end{array}$ & $\begin{array}{l}\operatorname{miRNA}-185 \\
\text { and } \\
\text { miRNA-186 }\end{array}$ & $\begin{array}{l}\text { Anti- } \\
\text { fibrotic }\end{array}$ & COL5A1 & $\begin{array}{l}\text { Regulation of collagen } \\
\text { V expression and EMT }\end{array}$ & $\begin{array}{l}\text { Down } \\
\text { regulated }\end{array}$ & $\begin{array}{l}\text { Lei et al., } \\
2016\end{array}$ \\
\hline XIII & $\begin{array}{l}\text { Human lung } \\
\text { fibrotic samples }\end{array}$ & $\begin{array}{l}\text { miRNA-130b- } \\
3 p\end{array}$ & $\begin{array}{l}\text { Anti- } \\
\text { fibrotic }\end{array}$ & IGF-1 & $\begin{array}{l}\text { Regulation of } \\
\text { fibroblasts activation } \\
\text { and EMT }\end{array}$ & $\begin{array}{l}\text { Down } \\
\text { regulated }\end{array}$ & $\begin{array}{l}\text { Li et al., } \\
2016\end{array}$ \\
\hline
\end{tabular}


Table 1. MiRNAs involved in the crucial signaling pathways of Idiopathic Pulmonary Fibrosis (Continued)

\begin{tabular}{|c|c|c|c|c|c|c|c|}
\hline & Sample & miRNA & $\begin{array}{l}\text { Function in } \\
\text { health }\end{array}$ & $\begin{array}{l}\text { Target of } \\
\text { action }\end{array}$ & Effect & $\begin{array}{l}\text { Status in } \\
\text { IPF }\end{array}$ & Reference \\
\hline XIV & $\begin{array}{l}\text { Human lung } \\
\text { fibrotic samples } \\
\text { and bleomycin } \\
\text { murine model }\end{array}$ & miRNA-101 & $\begin{array}{l}\text { Anti- } \\
\text { fibrotic }\end{array}$ & $\begin{array}{l}\text { Wnt and Smad } \\
2 / 3\end{array}$ & $\begin{array}{l}\text { Regulation of } \\
\text { fibroblasts } \\
\text { proliferation }\end{array}$ & $\begin{array}{l}\text { Down } \\
\text { regulated }\end{array}$ & $\begin{array}{l}\text { Huang } \\
\text { et al., } 2017\end{array}$ \\
\hline $\mathrm{XV}$ & $\begin{array}{l}\text { Human lung } \\
\text { fibrotic tissue and } \\
\text { bleomycine murine } \\
\text { model }\end{array}$ & $\begin{array}{l}\text { miRNA-323a- } \\
3 p\end{array}$ & $\begin{array}{l}\text { Anti- } \\
\text { fibrotic }\end{array}$ & Smad2 & $\begin{array}{l}\text { Regulation of } \\
\text { fibroblasts proliferation }\end{array}$ & $\begin{array}{l}\text { Down } \\
\text { regulated }\end{array}$ & $\begin{array}{l}\text { Ge at al., } \\
2016\end{array}$ \\
\hline XVI & $\begin{array}{l}\text { Human lung } \\
\text { fibrotic samples } \\
\text { and } A 549 \text { cells }\end{array}$ & miRNA-221 & $\begin{array}{l}\text { Anti- } \\
\text { fibrotic }\end{array}$ & HMGA2 & EMT regulation & $\begin{array}{l}\text { Down } \\
\text { regulated }\end{array}$ & $\begin{array}{l}\text { Wang et al., } \\
2016\end{array}$ \\
\hline XVII & $\begin{array}{l}\text { Bleomycin murine } \\
\text { model and TGF- } \beta \\
\text { treated human } \\
\text { embryonic } \\
\text { lung fibroblasts }\end{array}$ & miRNA-182-5p & Pro-fibrotic & $\begin{array}{l}\text { Smad2 and } \\
\text { Smad7 }\end{array}$ & $\begin{array}{l}\text { Increases the } \\
\text { expression of } \\
\text { profibrotic proteins } \\
\text { such as fibronectin, } \\
\alpha \text {-SMA, Smad2 and } \\
\text { Smad3 } \\
\text { Inhibition of Smad7 }\end{array}$ & $\begin{array}{l}\text { Up } \\
\text { regulated }\end{array}$ & $\begin{array}{l}\text { Chen et al, } \\
2019\end{array}$ \\
\hline XVIII & $\begin{array}{l}\text { Bleomycin murine } \\
\text { model and human } \\
\text { lung fibroblast }\end{array}$ & miRNA-21 & Pro-fibrotic & ADAMTS $\bowtie 1$ & $\begin{array}{l}\text { Promotes interstitial } \\
\text { fibroblasts proliferation } \\
\text { and increase } \\
\text { deposition of ECM }\end{array}$ & $\begin{array}{l}\text { Up } \\
\text { regulated }\end{array}$ & $\begin{array}{l}\text { Zhou et al., } \\
2018\end{array}$ \\
\hline XIX & $\begin{array}{l}\text { Bleomycin murine } \\
\text { model }\end{array}$ & miRNA-410 & Pro-fibrotic & $\begin{array}{l}\text { TGF- } \beta / \\
\text { ADAMTS1 } \\
\text { pathway }\end{array}$ & $\begin{array}{l}\text { Increases extracellular } \\
\text { matrix proteins } \\
\text { deposition and } \\
\text { fibroblasts proliferation }\end{array}$ & $\begin{array}{l}\text { Up } \\
\text { regulated }\end{array}$ & $\begin{array}{l}\text { Liu et al., } \\
2018\end{array}$ \\
\hline$x X$ & $\begin{array}{l}\text { Human lung } \\
\text { fibroblasts }\end{array}$ & miRNA-424 & Pro-fibrotic & Slit2 & $\begin{array}{l}\text { Positive feed-back } \\
\text { regulation of TGF-ß- } \\
\text { induced expression of } \\
\text { myofibroblast } \\
\text { differentiation markers } \\
\text { including } \alpha \\
\text {-SMA and CTGF }\end{array}$ & $\begin{array}{l}\text { Up } \\
\text { regulated }\end{array}$ & Huang 2020 \\
\hline XXI & A549 cells & miRNA-31 & Pro-fibrotic & $\begin{array}{l}\text { Smad2, Smad4 } \\
\text { and Smad6 }\end{array}$ & $\begin{array}{l}\text { Promotes } \\
\text { profibrotic factors } \\
\text { expression like MMP7 } \\
\text { Inhibits the expression } \\
\text { of Smad6 }\end{array}$ & $\begin{array}{l}\text { Up } \\
\text { regulated }\end{array}$ & $\begin{array}{l}\text { Wang et al, } \\
2019\end{array}$ \\
\hline \multicolumn{8}{|c|}{ MiRNAs involved in the EMT in a non Smad-dependent pathway } \\
\hline XXII & $\begin{array}{l}\text { TGF- } \beta \text { treated } \\
\text { human lung } \\
\text { fibroblasts }\end{array}$ & miRNA-340-5p & $\begin{array}{l}\text { Anti- } \\
\text { fibrotic }\end{array}$ & $\begin{array}{l}\text { p38/ } \\
\text { ATF1 signaling } \\
\text { axis }\end{array}$ & $\begin{array}{l}\text { Reduces extracellular } \\
\text { matrix deposition, } \\
\text { fibroblasts markers } \\
\text { such as } \\
\alpha \text {-SMA and COL1 and } \\
\text { reduces fibroblasts } \\
\text { proliferation, } \\
\text { and viability }\end{array}$ & $\begin{array}{l}\text { Up } \\
\text { regulated }\end{array}$ & $\begin{array}{l}\text { Wei et al., } \\
2020\end{array}$ \\
\hline XXIII & $\begin{array}{l}\text { Bleomycin murine } \\
\text { model }\end{array}$ & miRNA®448 & $\begin{array}{l}\text { Anti- } \\
\text { fibrotic }\end{array}$ & JNK & $\begin{array}{l}\text { Increased } \\
\text { cellular apoptosis, } \\
\text { decrease of cell } \\
\text { viability and reduced } \\
\text { collagen synthesis } \\
\text { by fibroblasts }\end{array}$ & $\begin{array}{l}\text { Up } \\
\text { regulated }\end{array}$ & $\begin{array}{l}\text { Xu et al., } \\
2020\end{array}$ \\
\hline XXIV & $\begin{array}{l}\text { Lipopolysaccharide } \\
\text { fibrosis murine } \\
\text { model }\end{array}$ & miRNA-506 & $\begin{array}{l}\text { Anti- } \\
\text { fibrotic }\end{array}$ & $\begin{array}{l}\text { P65 (NF-KB } \\
\text { subunit) }\end{array}$ & $\begin{array}{l}\text { Decreased } \\
\text { fibroblasts proliferation } \\
\text { and EMT }\end{array}$ & $\begin{array}{l}\text { Down } \\
\text { regulated }\end{array}$ & $\begin{array}{l}\text { Zhu et al., } \\
2019\end{array}$ \\
\hline
\end{tabular}


Table 1. MiRNAs involved in the crucial signaling pathways of Idiopathic Pulmonary Fibrosis (Continued)

\begin{tabular}{|c|c|c|c|c|c|c|c|}
\hline & Sample & miRNA & $\begin{array}{l}\text { Function in } \\
\text { health }\end{array}$ & $\begin{array}{l}\text { Target of } \\
\text { action }\end{array}$ & Effect & $\begin{array}{l}\text { Status in } \\
\text { IPF }\end{array}$ & Reference \\
\hline \multicolumn{8}{|c|}{ MiRNAs involved in the EMT involved in other signaling pathways } \\
\hline XXV & $\begin{array}{l}\text { Bleomycin murine } \\
\text { model }\end{array}$ & $\begin{array}{l}\text { miRNA-152- } \\
\text { 3p, miRNA- } \\
\text { 140-3p, } \\
\text { miRNA-148 } \\
\text { b-3p, and } \\
\text { miRNA-7a-5p } \\
\\
\text { miRNA-34a-5p, } \\
\text { miRNA-27 } \\
\text { b-3p, } \\
\text { miRNA-323- } \\
\text { 3p, miRNA- } \\
\text { 27a-3p, } \\
\text { miRNA- } \\
\text { 34c-5p, } \\
\text { miRNA-128- } \\
\text { 3p, and } \\
\text { miRNA-224-5p }\end{array}$ & $\begin{array}{l} \\
\text { Anti- } \\
\text { fibrotic }\end{array}$ & $\overline{\operatorname{lng} 5 \text { gene }}$ & $\begin{array}{l}\text { EMT induction by } \\
\text { downregulation of } \\
\text { TGF- } \beta \text { and Wnt/ } \beta \text { - } \\
\text { catenin pathways } \\
\\
\text { EMT induction } \\
\text { Through activation of } \\
\text { PI3K/AKT and Wnt/ } \beta- \\
\text { catenin pathways }\end{array}$ & $\begin{array}{l}\overline{\text { Down }} \\
\text { regulated }\end{array}$ & $\begin{array}{l}\text { Wang et al., } \\
2020\end{array}$ \\
\hline XXVI & $\begin{array}{l}\text { Human BAL and } \\
\text { THP-1 cells }\end{array}$ & miRNA-185 & $\begin{array}{l}\text { Anti- } \\
\text { fibrotic }\end{array}$ & $\begin{array}{l}\text { AKT and } \\
\text { COL1A1 } \\
\text { activation } \\
\text { through the } \\
\text { down } \\
\text { regulation of } \\
\text { miR-29 }\end{array}$ & $\begin{array}{l}\text { Decreased collagen } \\
\text { expression and } \\
\text { extracellular matrix } \\
\text { deposition }\end{array}$ & $\begin{array}{l}\text { Down } \\
\text { regulated }\end{array}$ & $\begin{array}{l}\text { Tsitoura } \\
\text { et al., } 2016\end{array}$ \\
\hline XXVII & $\begin{array}{l}\text { Bleomycin in vitro } \\
\text { model (MLE-12 cell } \\
\text { line) }\end{array}$ & miRNA-142-3p & $\begin{array}{l}\text { Anti- } \\
\text { fibrotic }\end{array}$ & $\begin{array}{l}\mathrm{PI} 3 \mathrm{~K} / \mathrm{AKT} / \\
\mathrm{mTOR}\end{array}$ & $\begin{array}{l}\text { Decreased cellular } \\
\text { apoptosis and } \\
\text { increase of of IL-1 and } \\
\text { TNF- } \alpha \text { levels }\end{array}$ & $\begin{array}{l}\text { Down } \\
\text { regulated }\end{array}$ & $\begin{array}{l}\text { Guo et al., } \\
2017\end{array}$ \\
\hline XXVIII & $\begin{array}{l}\text { Mice and human } \\
\text { lung tissue } \\
\text { samples }\end{array}$ & miRNA-29c & $\begin{array}{l}\text { Anti- } \\
\text { fibrotic }\end{array}$ & Foxo3a & $\begin{array}{l}\text { Decreased cellular } \\
\text { apoptosis }\end{array}$ & $\begin{array}{l}\text { Down } \\
\text { regulated }\end{array}$ & $\begin{array}{l}\text { Xie et al., } \\
201\end{array}$ \\
\hline XXIX & $\begin{array}{l}\text { Bleomycin murine } \\
\text { model }\end{array}$ & miRNA-124-3p & Pro fibrotic & AXIN1 & $\begin{array}{l}\text { TGF- } \beta 1 \text { induced } \\
\text { fibroblasts } \\
\text { differentiation and } \\
\text { increased Wnt } \\
\text { pathway }\end{array}$ & $\begin{array}{l}\text { Up } \\
\text { regulated }\end{array}$ & $\begin{array}{l}\text { Lu et al., } \\
2019\end{array}$ \\
\hline XXX & $\begin{array}{l}\text { Bleomycin murine } \\
\text { model and human } \\
\text { fibrotic lung } \\
\text { sample }\end{array}$ & miRNA-301 & Pro-fibrotic & $\begin{array}{l}\mathrm{TSC} 1 / \mathrm{mTOR} \\
\text { pathway }\end{array}$ & $\begin{array}{l}\text { Promotes fibroblasts } \\
\text { activation, proliferation } \\
\text { and trans } \\
\text { differentiation into } \\
\text { myofibroblasts and } \\
\text { collagen deposition }\end{array}$ & $\begin{array}{l}\text { Up } \\
\text { regulated }\end{array}$ & $\begin{array}{l}\text { Wang et al., } \\
2020\end{array}$ \\
\hline
\end{tabular}

miRNA: microRNA; TGFBRI: type I TGF- $\beta$ receptor; TGFBRII: type II TGF- $\beta$ receptor; $\alpha$-SMA: alpha-smooth muscle actin ; TGF- $\beta 1$ : transforming growth factor beta 1 ; CTGF: connective tissue growth factor; PI3K-AKT-FOX03a-mTOR: phosphatidylinositol 3-kinase, protein kinase B, forkhead box 03, mammalian target of rapamycin; NF-kb: nuclear factor kappa-light-chain-enhancer of activated B cells; ZEB 1 / 2: zinc finger E-Box binding homeobox 1 and 2; EMT: epithelial mesenchymal transition; IGF-1: insulin-like growth factor-1; Wnt: wingless and int-1; HMGA2: high-mobility group AT-hook2; ADAMTS-1: metallopeptidase with thrombospondin type motif 1; ECM: extracellular matrix; MMP: matrix metallopeptidase; JNK: c-Jun N-terminal kinase; KIf4: kruppel-like factor 4; Ing5: growth family member5; BAL: bronchoalveolar lavage; IL1: interleukin 1; TNF- $\alpha$ : tumor necrosis factor alpha; AXIN: axis inhibition protein 1; TSC1: tuberous sclerosis complex.

bleomycin murine model, miRNA-101 acts via the Wnt and TGF- $\beta / S$ mad2/3 pathways but its downregulated status generates an increase in fibroblasts proliferation, collagen synthesis and elevation of $\alpha-S_{M A}^{30}$ and
miRNA-323a-3p is not able to regulate Smad2/3 complex therefore it cannot suppress fibroblast differentiation and expression of matrix proteins. ${ }^{31}$ MiRNA-221 is another one studied on human lung fibrotic samples 
but also in A549 cells and it was seen that it cannot regulate EMT process because it not able to regulate HMGA2 ${ }^{32}$.

Finally, there are some miRNAs which have a pro fibrotic role due to their up regulation such as miRNA-1825p, miRNA-21, miRNA-410, miRNA-424 and miRNA-31.

MiRNA-182-5p and miRNA-21 have been studied in a bleomycin murine model but also in human lung fibroblasts and it was found that miRNA-182-5p has a direct effect stimulating on Smad2/3 complex and inhibiting Smad7 $7^{33}$ and miRNA-21 causes an abnormal deposition of ECM through the increase of TGF- $\beta$ / ADAMTS-1 signal pathway and inhibition of Smad7 to ${ }^{34}$. MiRNA-410 was studied in a bleomycin model and is involved in the activation of TGF- $\beta 1 /$ ADAMTS-1 signaling pathway as well and it has a promising therapeutic future because through the inhibition of this miRNA there would be a regulation of extracellular matrix proteins like COL1 and COL3 and fibroblasts proliferation ${ }^{35}$.

In some recent studies, there has been found that miRNA-424 has an up regulated status in human lung fibroblasts due to Slit2 inhibition which generates a positive feed-back regulation of the TGF- $\beta$-induced expression of myofibroblast differentiation markers including $\alpha$-SMA and $\mathrm{CTGF}^{36}$. and miRNA-31 promotes Smad2 and Smad4 heterodimers formation and inhibits Smad6 in A 549 cells $^{20}$.

\section{miRNAs involved in EMT in a non Smad- dependent way}

In recent studies it has been demonstrated that there are some miRNAs that act on non-Smad dependent pathways with an antifibrotic role like miRNA-340-5p, miRNA-448 and miRNA-506.

MiRNA-340-5 $p$ which is upregulated in TGF- $\beta$ treated human lung fibroblasts regulates the ATF1/p38 signaling axis that reduces extracellular matrix, fibroblasts markers such as $\alpha$-SMA and COL-1and reduces fibroblasts proliferation ${ }^{37}$.

MiRNA-448 studied in mice fibrotic tissue, was found to be upregulated and its function is to suppress the JNK signaling pathway that can inhibit IPF progression by downregulating cell proliferation and collagen synthesis by promoting apoptosis in fibroblasts ${ }^{38}$.

On the other hand, miRNA-506 has an antifibrotic role through the inhibition of $p 65$ which is a subunit of $\mathrm{NF}-\mathrm{KB}$, however its downregulated status in a fibrosis murine model promotes fibroblasts recruitment, EMT and decreases fibroblast apoptosis ${ }^{39}$.

\section{MiRNAs involved in EMT through other signaling pathways}

There are some several miRNAs that have an antifibrotic role, however, due to their down regulated status in lung fibrosis they are not able to fulfill their role.

For example, miRNA-152-3p, miRNA-140-3p, miRNA-148 b-3p, and miRNA-7a-5p studied in bleomycin murine model, are not able to downregulate Kruppel-like factor 4 (Klf4), a negative regulator of $\alpha$-SMA which has a direct effect on SNAI2, therefore suppressing TGF $\beta 1$-induced EMT and they are not able to regulate the Wnt/ $\beta$ - catenin pathway either. Also, miRNA34a-5p, miRNA-27 b-3p, miRNA-323-3p, miRNA-27a-3p, miRNA-34c-5p, miRNA-128-3p, and miRNA-224-5p cannot regulate the growth family member5 (Ing5) which regulates PI3K/AKT and $\mathrm{Wnt} / \beta$-catenin pathway therefore there is an increment in the fibrosis process ${ }^{40}$.

MiRNA-185 downregulation in human bronchoalveolar lavage (BAL) contributes to collagen deposition due to AKT overactivation and miRNA-29a downregulation which causes the overexpression of COL1A1 gene, therefore this miRNA has been proposed as possible diagnosis biomarkers for patients with IPF ${ }^{41}$. Moreover, with miRNA-142-3p mimic transfection, in bleomycin-induced injuries in an in vitro IPF model it was possible to alleviate cell injury and production of pro-inflammatory factors through the regulation of $\mathrm{PI} 3 \mathrm{~K} / \mathrm{AKT}$, which indicated the protective role of this miRNA, however, even though it has a anti fibrotic role it is diminished in a fibrosis state ${ }^{42}$. Another example is miRNA-29c which have an impact on cell apoptosis of AECS2 because its function is to regulate $\mathrm{FoxO} 3 \mathrm{a}$, however, this miRNA is negatively regulated in human lung tissue resulting in a resistance to apoptosis and lack of regulation of ECM-related target genes such as COL3A $A^{43}$.

Finally, there are some miRNAs which have a pro fibrotic role due to their up regulation such as miRNA124-3p and miRNA-301a, both have been studied on a bleomycin model and miRNA-301a has also been studied on human lung fibrotic tissue samples.

MiRNA-124-3p plays an important role in TGF- $\beta 1$ induced fibrogenic cell differentiation through the regulation of AXIN1 protein, which is involved in the activation of Wnt pathway ${ }^{44}$ and miRNA-301a promotes the up regulation of TSC1 gene which acts through the mTOR signaling pathway promoting fibroblasts activation and has been proposed as a possible therapeutic target ${ }^{45}$. 


\section{Results}

After reviewing fifty original articles about MiRNAs involved in idiopathic pulmonary fibrosis, it was decided to include only those that related miRNAs to the main signaling pathways involved in the fibrotic process of lung parenchyma, such as TGF- $\beta$, Wnt $/ \beta$-catenin and PI3K-Akt-FOXO3a-mTOR pathways.

MiRNAs are short, non-coding RNAs with an extension of approximately 21-23 nucleotides which play an important role in the genesis and development of IPF thanks to their supra regulated state by favoring epithelial mesenchymal transition and fibroblasts stimulation with the consequent synthesis of extracellular matrix proteins such as collagen; however, there is another pro fibrotic mechanism in which miRNAs cannot exert their antifibrotic effect because they are in an infra regulated state, which generates an inadequate control of intracellular receptors of the signaling pathways which will favor lung fibrosis.

Among these signaling pathways there is the TGF- $\beta$ pathway, which exert its function through a Smad and non Smad dependent way. It is the main pathway involved in the regulation of the expression of profibrotic genes as well as the differentiation and proliferation of fibroblasts and the consequent deposition of collagen. There are also other alternative pathways such as the Wnt/ $\beta$-catenin pathway and the PI3K-Akt-FOXO3amTOR pathway, which are also stimulated by miRNAs, and they related to alveolar repair through fibroblasts proliferation and extracellular matrix proteins production which generates pulmonary fibrosis.

\section{Discussion}

IPF is a progressive and degrading interstitial lung disease with poor prognosis and few therapeutic options. Nowadays it has been proposed that several miRNAs play an important role in the pathogenesis of this disease, both in human and murine fibrosis models, because they are able to regulate cellular processes such as cellular apoptosis, proliferation, and differentiation through the stimulation of different receptors within signaling pathways which causes a remodeling effect of lung parenchyma.

In this review the current knowledge about these miRNAs was grouped and synthesized to have a better understanding about their different mechanisms of action within the main signaling pathways which have as common end the trans differentiation of fibroblasts into myofibroblasts and the synthesis of extracellular matrix proteins that contribute with the fibrotic process.

\section{Conclusions}

MiRNAs have been studied in IPF, to expand the knowledge about the pathogenesis of this disease but also, they have been postulated as a new therapeutic option because nowadays the treatment of this disease is not very effective and is not curative either, so if it is possible to modify or block the action of a specific miRNA it could generate a change in the mechanism of the disease. Although it is not yet known whether this therapy will have adverse effects, it is believed that therapy based on miRNAs could be the basis of an effective treatment for pulmonary fibrosis since there is a precedent regarding the possible utility of miRNAs as therapeutic agents. This has been studied in different types of cancer where in which it is used miRNA mimic therapy and viral vectors containing the miRNA target to restore the down regulated status of anti fibrotic miRNAs and anti-miRNAs to block the pro fibrotic role of supra regulated miRNAs. However, it is suggested to expand the studies about miRNAs involved in IPF to continue updating information about different mechanisms of action altered in this pathology so that in the future, being able to help patients suffering from this chronic and devasting disease by proposing a new therapeutic agent more effective than those existing today and to provide a better quality of life.

\section{Acknowledgements}

The INER is thanked for being the source of inspiration for this article.

\section{Funding}

There was no funding for this article.

\section{Conflict of interests}

The authors declare that they have no conflict of interest.

\section{Ethical disclosures}

Protection of human and animal subjects. The authors declare that no experiments were performed on humans or animals for this study.

Confidentiality of data. The authors declare that no patient data appear in this article. 
Right to privacy and informed consent. The authors declare that no patient data appear in this article.

\section{References}

1. Selman M, Pardo A. The leading role of epithelial cells in the pathogenesis of idiopathic pulmonary fibrosis. Cell Signal. 2020; 66:109482

2. Nemeth J, Schundner A, Quast K, Winkelmann VE and Frick M. A Novel Fibroblast Reporter Cell Line for in vitro Studies of Pulmonary Fibrosis. Front. Physiol. 2020; 11:567675

3. Calin GA, Sevignani C, Dumitru CD, Hyslop T, Noch E, Yendamuri S, et al. Human microRNA genes are frequently located at fragile sites and genomic regions involved in cancers. Proc Natl Acad Sci USA. 2004;101(9):2999-3004

4. Thiery JP, Acloque $H$, Huang RY, Nieto MA. Epithelial-mesenchymal transitions in development and disease. Cell. 2009;139(5):871-890

5. Nieto MA, Huang RY, Jackson RA, Thiery JP. EMT. Cell. 2016;166(1):21-45

6. Pandit KV, Milosevic J. MicroRNA regulatory networks in idiopathic pulmonary fibrosis. Biochem Cell Biol. 2015;93(2):129-137

7. Bourd-Boittin K, Bonnier D, Leyme A, Mari B, Tuffery P, Samson M, et al. Protease profiling of liver fibrosis reveals the ADAM metallopeptidase with thrombospondin type 1 motif, 1 as a central activator of transforming growth factor beta. Hepatology. 2011;54(6):2173-84.

8. Rajasekaran S, Rajaguru P, Sudhakar Gandhi PS. MicroRNAs as potential targets for progressive pulmonary fibrosis. Front. Pharmacol. 2015; 6:254

9. Chanda D, Otoupalova E, Smith SR, Volckaert T, De Langhe SP, Thannickal VJ. Developmental pathways in the pathogenesis of lung fibrosis. Mol Aspects Med. 2019; 65:56-69.

10. Kang H. Role of MicroRNAs in TGF- $\beta$ Signaling Pathway-Mediated Pulmonary Fibrosis. Int J Mol Sci. 2017;18(12):2527.

11. Lifshitz V, Frenkel D. Handbook of Biologically Active Peptides. Academic Press. Loa Angeles., United States of America, 2013

12. Derynck, R, Zhang Y. Smad-dependent and Smad-independent pathways in TGF- $\beta$ family signalling. Nature 2003; 425:577-584

13. Skronska-Wasek W, Gosens R, Königshoff M, Baarsma HA. WNT receptor signalling in lung physiology and pathology. Pharmacol Ther. 2018; 187:150-166

14. Königshoff, M, Balsara, N, Pfaff, EM, Kramer M, Chrobak, I, Seeger W, et al. Functional Wnt signaling is increased in idiopathic pulmonary fibrosis. PloS one. 2008;3(5): e2142

15. Lu Y, Azad N, Wang L, lyer AK, Castranova V, Jiang BH, et al. Phosphatidylinositol-3-kinase/akt regulates bleomycin-induced fibroblast proliferation and collagen production. Am J Respir Cell Mol Biol. 2010; 42:432-441

16. Laplante M, Sabatini DM. mTOR signaling in growth control and disease. Cell. 2012;149(2):274-293

17. Wang C, Gu S, Cao H, Li Z, Xiang Z, Hu, K, et al. miR-877-3p targets Smad7 and is associated with myofibroblast differentiation and bleomycin-induced lung fibrosis. Sci Rep. 2016; 6:30122

18. Stolzenburg LR, Wachtel S, Dang H, Harris A. miR-1343 attenuates pathways of fibrosis by targeting the TGF- $\beta$ receptors. Biochem J. 2016;473(3):245-256

19. Wei P, Xie Y, Abel PW, Huang Y, Ma Q, Li L, et al. Transforming growth factor (TGF)- $\beta 1$-induced miR-133a inhibits myofibroblast differentiation and pulmonary fibrosis. Cell Death Dis. 2019;10(9):670

20. Wang CJ, Li BB, Tan YJ, Zhang GM, Cheng GL, Ren YS. MicroRNA-31/184 is involved in transforming growth factor- $\beta$-induced apoptosis in A549 human alveolar adenocarcinoma cells. Life Sci. 2020; 242:117205

21. Chi L, Xiao Y, Zhu L, Zhang M, Xu B, Xia H, et al. microRNA-155 attenuates profibrotic effects of transforming growth factor-beta on human lung fibroblasts. J Biol Regul Homeost Agents. 2019;33(5):1415-1424

22. Cui H, Banerjee S, Xie N, Ge J, Liu R.M, Matalon S, et al. MicroRNA-27a-3p Is a Negative Regulator of Lung Fibrosis by Targeting Myofibroblast Differentiation. Am J Respir Cell Mol Biol. 2016;54(6):843-852

23. Souma K, Shichino S, Hashimoto S, Ueha S, Tsukui T, Nakajima T, et al. Lung fibroblasts express a miR-19a-19b-20a sub-cluster to suppress TGF- $\beta$-associated fibroblast activation in murine pulmonary fibrosis. Sci Rep. 2018;8(1):16642
24. Yamada Y, Takanashi M, Sudo K, Ueda S, Ohno SI, Kuroda M, et al. Novel form of miR-29b suppresses bleomycin-induced pulmonary fibrosis. PLoS One. 2017;12(2): e0171957

25. Zhang $Q$, Ye $H$, Xiang F, Song LJ, Zhou LL, Cai PC, et al. miR-18a-5p Inhibits Sub-pleural Pulmonary Fibrosis by Targeting TGF- $\beta$ Receptor II. Mol Ther. 2017;25(3):728-738.

26. Cao Y, Liu Y, Ping F, Yi L, Zeng Z, Li Y, et al. miR-200b/c attenuates lipopolysaccharide-induced early pulmonary fibrosis by targeting ZEB1/2 via p38 MAPK and TGF- $\beta /$ smad3 signaling pathways. Lab Invest. 2018;98(3):339-359

27. Zeng $X$, Huang $C$, Senavirathna L, Wang $P$, Liu L, et al. miR-27b inhibits fibroblast activation via targeting TGF $\beta$ signaling pathway. BMC Cell Biol. 2017; 18(1):9

28. Lei GS, Kline HL, Lee CH, Wilkes DS, Zhang C, et al. MiR-185 and miR186 during Idiopathic Pulmonary Fibrosis. Am J Pathol. 2016;186(9):2310-2316

29. Li S, Geng J, Xu X, Huang X, Leng D, Jiang D, et al. MiR-130b-3p Modulates Epithelial-Mesenchymal Crosstalk in Lung Fibrosis by Targeting IGF-1. PLoS One. 2016;11(3): e0150418.

30. Huang C, Xiao X, Yang Y, Mishra A, Liang Y, Zeng X, et al. MicroRNA-101 attenuates pulmonary fibrosis by inhibiting fibroblast proliferation and activation. J Biol Chem. 2019 Apr 26;294(17):6694]. J Biol Chem. 2017;292(40):16420-16439

31. Ge L, Habiel DM, Hansbro PM, Kim RY, Gharib SA, Edelman JD, et al. MiR-323a-3p regulates lung fibrosis by targeting multiple profibrotic pathways. JCl Insight. 2016;1(20): e90301

32. Wang YC, Liu JS, Tang HK, Nie J, Zhu JX, Wen LL, et al. miR221 targets HMGA2 to inhibit bleomycininduced pulmonary fibrosis by regulating TGF $\beta 1 / S m a d 3-i n d u c e d$ EMT. Int J Mol Med. 2016;38(4):1208-1216

33. Chen $Y$, Zhang Q, Zhou Y, Yang Z, Tan M. Inhibition of miR-182-5p attenuates pulmonary fibrosis via TGF- $\beta / S m a d$ pathway. Hum Exp Toxicol. 2020;39(5):683-695

34. Zhou J, Xu Q, Zhang Q, Wang Z, Guan S. A novel molecular mechanism of microRNA-21 inducing pulmonary fibrosis and human pulmonary fibroblast extracellular matrix through transforming growth factor $\beta 1$-mediated SMADs activation. J Cell Biochem. 2018;119(9):7834-7843

35. Liu H, He Y, Jiang Z, Shen S, Mei J, Tang M. Prodigiosin Alleviates Pulmonary Fibrosis Through Inhibiting miRNA-410 and TGF- $\beta 1 /$ ADAMTS-1 Signaling Pathway. Cell Physiol Biochem. 2018;49(2):501-511

36. Huang $Y$, Xie $Y$, Abel PW, Wei P, Plowman J, Toews ML, et al. TGF- $\beta 1$-induced miR-424 promotes pulmonary myofibroblast differentiation by targeting Slit2 protein expression. Biochem Pharmacol. 2020; $180: 114172$.

37. Wei YQ, Guo YF, Yang SM, Ma HH, Li J. MiR-340-5p mitigates the proliferation and activation of fibroblast in lung fibrosis by targeting TGF- $\beta /$ p38/ATF1 signaling pathway. Eur Rev Med Pharmacol Sci. 2020;24(11):6252-6261

38. Xu F, Xu F, Xie S, Zuo W, Wen G, Zhao T, et al. MicroRNA-448 overexpression inhibits fibroblast proliferation and collagen synthesis and promotes cell apoptosis via targeting ABCC3 through the JNK signaling pathway. J Cell Physiol. 2020;235(2):1374-1385

39. Zhu M, An Y, Zhang X, Wang Z, Duan H. Experimental pulmonary fibrosis was suppressed by microRNA-506 through NF-kappa-mediated apoptosis and inflammation. Cell Tissue Res. 2019;378(2):255-265

40. Wang C, Cao H, Gu S, Shi C, Chen X, Han X. Expression analysis of microRNAs and mRNAs in myofibroblast differentiation of lung resident mesenchymal stem cells. Differentiation. 2020; 112:10-16

41. Tsitoura E, Wells AU, Karagiannis K, Lasithiotaki I, Vasarmidi E, Bibaki E, et al. MiR-185/AKT and miR-29a/collagen 1a pathways are activated in IPF BAL cells. Oncotarget. 2016;7(46):74569-74581

42. Guo F, Lin SC, Zhao MS, Yu B, Li XY, Gao Q, et al. microRNA-142-3p inhibits apoptosis and inflammation induced by bleomycin through down-regulation of Cox-2 in MLE-12 cells. Braz J Med Biol Res. 2017;50(7): e5974

43. Xie T, Liang J, Geng Y, Liu N, Kurkciyan A, Kulur V, et al. MicroRNA-29c Prevents Pulmonary Fibrosis by Regulating Epithelial Cell Renewal and Apoptosis. Am J Respir Cell Mol Biol. 2017;57(6):721-732

44. Lu Y, Zhang T, Shan S, Wang S, Bian W, Ren T, et al. MiR-124 regulates transforming growth factor- $\beta 1$ induced differentiation of lung resident mesenchymal stem cells to myofibroblast by repressing Wnt/B-catenin signaling. Dev Biol. 2019;449(2):115-121

45. Wang J, Li X, Zhong M, Wang Y, Zou L, Wang M, et al. MiR-301a Suppression within Fibroblasts Limits the Progression of Fibrosis through the TSC1/mTOR Pathway. Mol Ther Nucleic Acids. 2020; 21:217-228 